\title{
ARTICLE
}

\section{Black carbon absorption at the global scale is affected by particle-scale diversity in composition}

\author{
Laura Fierce ${ }^{1,2}$, Tami C. Bond ${ }^{3}$, Susanne E. Bauer ${ }^{4,5}$, Francisco Mena ${ }^{3}$ \& Nicole Riemer ${ }^{6}$
}

Atmospheric black carbon ( $B C$ ) exerts a strong, but uncertain, warming effect on the climate. $B C$ that is coated with non-absorbing material absorbs more strongly than the same amount of $\mathrm{BC}$ in an uncoated particle, but the magnitude of this absorption enhancement $\left(E_{\mathrm{abs}}\right)$ is not well constrained. Modelling studies and laboratory measurements have found stronger absorption enhancement than has been observed in the atmosphere. Here, using a particleresolved aerosol model to simulate diverse $B C$ populations, we show that absorption is overestimated by as much as a factor of two if diversity is neglected and population-averaged composition is assumed across all BC-containing particles. If, instead, composition diversity is resolved, we find $E_{\text {abs }}=1-1.5$ at low relative humidity, consistent with ambient observations. This study offers not only an explanation for the discrepancy between modelled and observed absorption enhancement, but also demonstrates how particle-scale simulations can be used to develop relationships for global-scale models.

\footnotetext{
${ }^{1}$ Department of Environmental and Climate Sciences, Brookhaven National Laboratory, Upton, New York 11973, USA. ${ }^{2}$ Visiting Scientists Program, University Corporation for Atmospheric Research, Boulder, Colorado 80307, USA. ${ }^{3}$ Department of Civil and Environmental Engineering, University of Illinois at UrbanaChampaign, Urbana, Illinois 61801, USA. ${ }^{4}$ NASA Goddard Institute for Space Studies, New York City, New York 10025, USA. ${ }^{5}$ The Earth Institute, Columbia University, New York City, New York 10025, USA. ${ }^{6}$ Department of Atmospheric Sciences, University of Illinois at Urbana-Champaign, Urbana, Illinois 61801, USA. Correspondence and requests for materials should be addressed to L.F. (email: Ifierce@bnl.gov).
} 
B lack carbon (BC) is the most strongly absorbing component of atmospheric aerosol ${ }^{1}$, leading to strong atmospheric warming. Simulations of the global atmosphere are used to estimate this warming. One challenge in predicting absorption per BC mass is accounting for the particle microphysical details that affect light absorption and radiative transfer within these large-scale models. Within individual particles, BC is mixed with other aerosol components to varying degree $^{2-4}$, depending on the emission source ${ }^{5-8}$ and the extent of atmospheric processing ${ }^{9-13}$. Modelled absorption by BC is strongly enhanced if $\mathrm{BC}$ is coated with non-absorbing aerosol components (for example, refs 14,15), but ambient observations show weaker levels of absorption enhancement ${ }^{15-17}$. To our knowledge, refs $15-17$ are the only studies that compare modelled and observed light absorption without significant measurement artifacts.

This discrepancy in modelled and observed light absorption indicates that the characteristics of BC-containing particles assumed in many models do not adequately represent BCcontaining particles found in the atmosphere. Treatments of particle composition vary between global models, from simple mass-based schemes ${ }^{18,19}$, which do not track any information about particle size or composition, to modal ${ }^{20-22}$ and sectional $^{23,24}$ schemes, which simulate the evolution of the particle size distribution but assume that particles within the same mode or of the same size have the same composition. Variants of these broad categories refine aspects of the particle population. For example, some sectional schemes represent separate particle sub-populations and track size-resolved composition within each population (for example, ref. 25). Because even the most sophisticated global models are not able to track characteristics of individual particles ${ }^{22,25}$, it has not been possible to explore how microscale particle details influence macroscale radiative effects.

This study investigates errors in modelled light absorption caused by ignoring diversity in particle composition, and shows that absorption is strongly affected by the distribution of components among individual particles. We apply a unique aerosol model that fully resolves the composition of individual particles, enabling us to evaluate how knowledge of particle-level composition impacts light absorption by $\mathrm{BC}$. In particular, we compare absorption enhancement by $\mathrm{BC}$ using particle-resolved composition and absorption enhancement by $\mathrm{BC}$ assuming the mass fraction of each aerosol component is the same for all particles. Absorption enhancement $\left(E_{\mathrm{abs}}\right)$ is defined as the absorption by mixed $\mathrm{BC}$-containing particles relative to absorption by the same amount of $\mathrm{BC}$ in pure, uncoated particles. While other studies show modelled absorption depends strongly on the representation of particle morphology (for example, refs 26-28) and on absorption by organic carbon coatings (for example, refs $16,17)$, our findings suggest that diversity in particle composition also plays an important role. We show that oversimplifying the representation of particle composition leads to overestimation in absorption enhancement, which is one factor affecting atmospheric heating by BC. To identify factors influencing light absorption by $\mathrm{BC}$, we performed a nonparametric regression on a series of simulations to identify the key independent variables that most affect $E_{\text {abs. }}$. Through this nonparametric analysis, we derived a relationship for $E_{\mathrm{abs}}$ as a function of the populationaveraged mass fraction of aerosol components and the environmental relative humidity (RH), such that the relationship for $E_{\text {abs }}$ accounts for particle-level variation in composition but depends only on variables that many global models already track. Finally, by applying this nonparametric relationship to output from a global model, we demonstrate that light absorption by $\mathrm{BC}$ at the global scale depends strongly on particle-scale diversity in composition.

\section{Results}

Absorption by BC in individual particles. The particle-resolved model PartMC-MOSAIC ${ }^{29,30}$ was used to simulate diversity in per-particle composition for populations of BC-containing particles. We considered 100 week-long simulations of urban air parcels, varying gas and particle emissions, background particle concentrations and meteorological conditions (Methods section). Although several particle types were included in the simulations, this study focuses on the evolution of the BC-containing particles only, which originate from combustion sources. These combustion particles were emitted as a mixture of $\mathrm{BC}$ and primary organic aerosol (POA), where the ratio of $\mathrm{BC}$ to POA varied among simulations to represent differences in the composition of emitted particles between BC sources. After their emission, the combustion particles accumulated additional coating through condensation of semi-volatile gases and coagulation with other particles.

In the particle-resolved representation of composition, each particle is different, depending on how recently it was emitted, its composition at the time it was emitted and the changes in composition that occurred since its emission. The wide variation in per-particle composition represented by the particle-resolved model is illustrated in Fig. 1a. Figure 1a shows a subset of particles sampled from a population of thousands of BC-containing particles in an aged air mass, sampled from a single scenario at 18:00 hours on the first day. Simulation settings for this scenario are outlined in Supplementary Table 1. The particle properties shown in Fig. 1a are sorted according to the mass of BC contained in each particle, which is shown in Fig. 1b. From this particle-resolved model output, absorption by each individual particle was modelled as a function of its composition, the number of $\mathrm{BC}$ inclusions that it contains and the environmental RH (Methods section). Rather than applying the approximation that $\mathrm{BC}$ exists as a single inclusion at the centre of each BC-containing particle, as in the widely-used core-shell approximation, we modelled particles using the dynamic effective medium approximation (DEMA) ${ }^{31,32}$. Under this effective medium approximation, each particle is assumed to contain one or more randomly-distributed BC inclusions that are fully encompassed in the non-absorbing material, including water, where changes in number of inclusions through coagulation events are tracked by the particle-resolved model. Water uptake by each particle was modelled as a function of particle-level composition and the environmental $\mathrm{RH}$ using the $\kappa$-Köhler model $^{33}$ (Methods section). We focus on absorption enhancement due to non-absorbing coatings and, therefore, do not consider absorption by organic carbon. Absorption enhancement by BC within individual particles is shown for $\mathrm{RH}=50 \%$ in Fig. 1c. Within the same population, particle-level absorption enhancement ranges from $\sim 1$ (no enhancement) to $>2$, depending on the particle's size and composition.

Absorption enhancement for the entire $\mathrm{BC}$ population is the sum over the absorption cross sections of coated particles divided by the sum of the absorption cross sections of uncoated particles. Absorption enhancement by the diverse population modelled by PartMC-MOSAIC is $\sim 1.3$ at $\mathrm{RH}=50 \%$ (solid line in Fig. 1e). Global aerosol models do not fully resolve this variation in particle composition. Instead, many aerosol models track one or more separate populations of BC-containing particles but assume uniform composition across all particles within each population $^{21,22,34}$. The average composition for the example population is shown in Fig. 1d. If the amount of $\mathrm{BC}$ in each particle is assumed to be the same as in the particle-resolved case but all BC-containing particles are assumed to have the average composition (Fig. 1d), we find $E_{\text {abs }}>2$ (dashed line in Fig. 1e), overestimating modelled absorption by $50 \%$ relative to absorption 
Particle-resolved composition
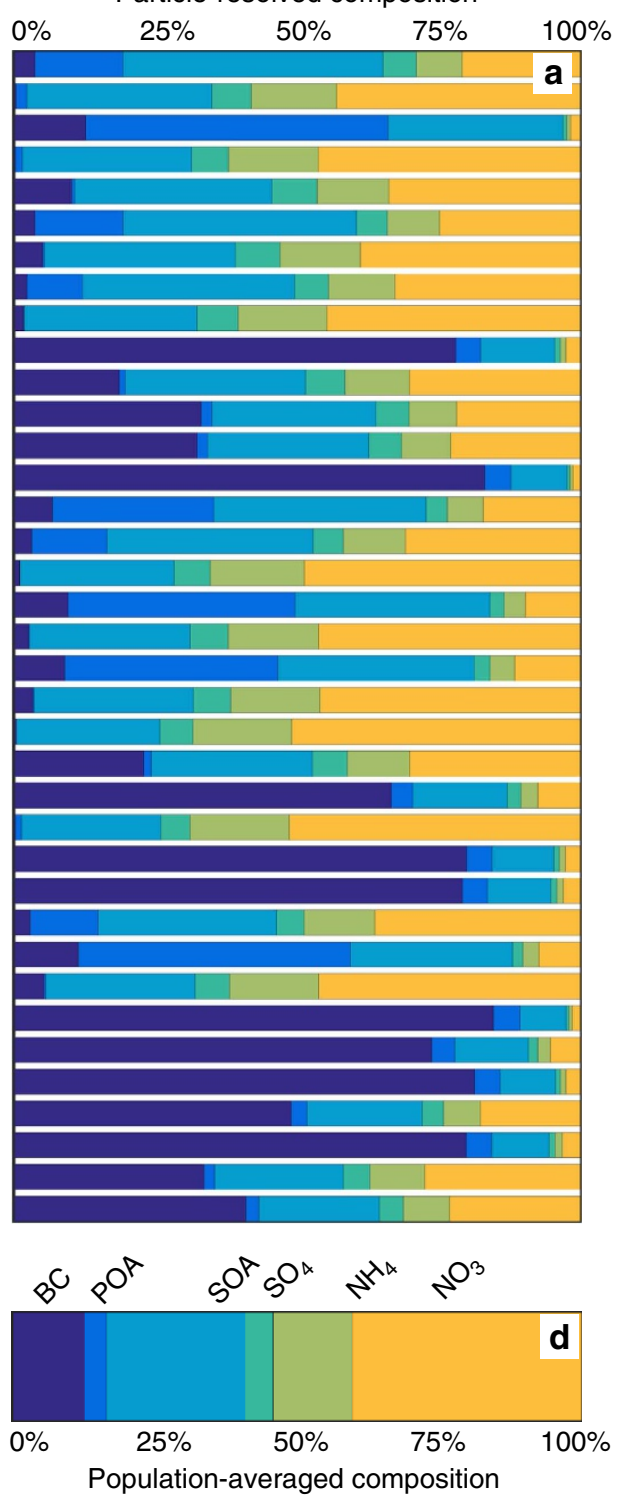

Per-particle

$\mathrm{BC}$ mass $(\mathrm{pg})$
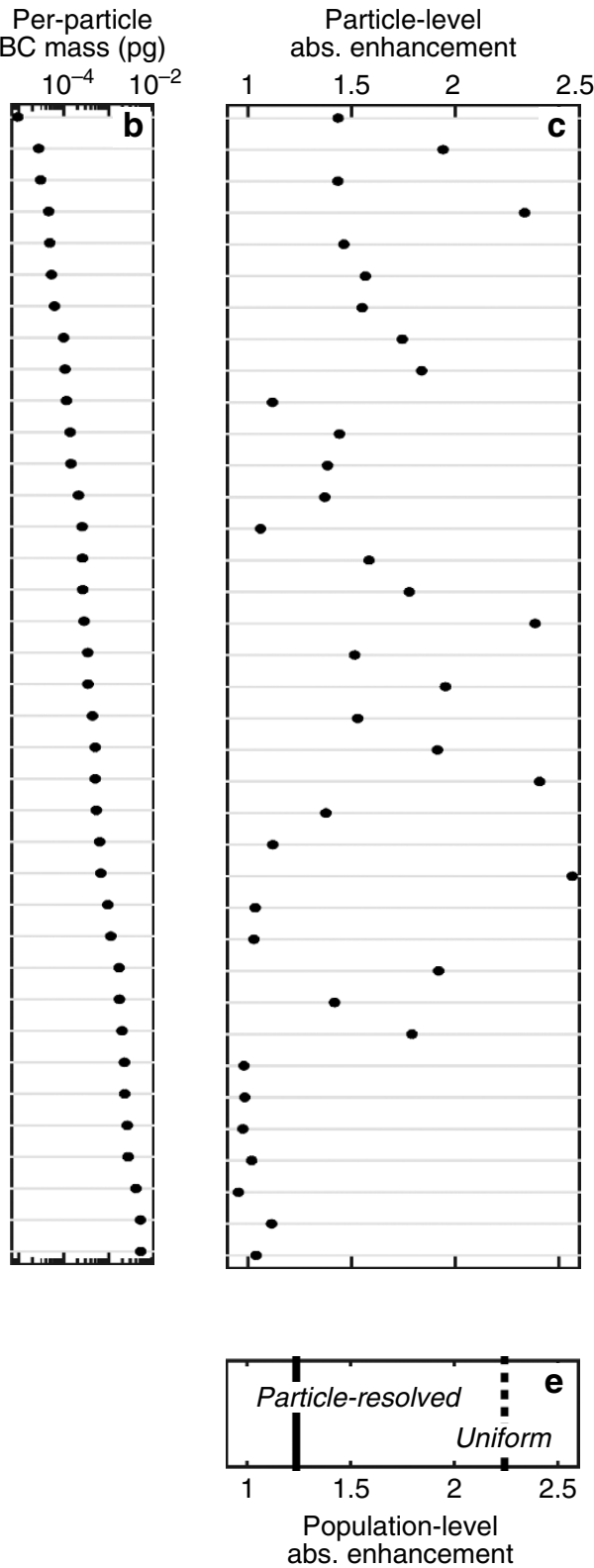

Figure 1 | Role of particle composition on absorption enhancement in baseline population. (a) Per-particle volume composition for subset of particles in a single population, (b) mass of BC contained in each particle and (c) the absorption enhancement by the BC within each particle, all shown for the particle-resolved representation, as well as the (d) volume composition averaged across all BC-containing particles. (e) Overall absorption enhancement by BC in the population is computed from particle-resolved composition (solid line) and, for the same population, assuming uniform composition (dashed line).

derived from particle-resolved output. Enhancement in light absorption due to radiative interactions between particles, which may also affect heating rates ${ }^{35}$, is not considered.

This error in modelled absorption from the simplified representation is caused by failure to capture the diversity in composition that is simulated by the particle-resolved model. While per-particle composition varies, even for particles of the same size, the mass fraction of coating material (Fig. 1a) tends to be smaller for particles that contain large amounts of BC (Fig. 1b), corresponding to weak enhancement in light absorption by these particles (Fig. 1c). Figure 2 confirms that, across the entire diverse $\mathrm{BC}$ population, most of the coating material is contained in particles that have small amounts of $\mathrm{BC}$ mass, while most of the $\mathrm{BC}$ mass resides in particles with large $\mathrm{BC}$ inclusions and only thin coatings due to variation in coating accumulation rates as a function of particle size. For comparison, Fig. 2 also shows how the aerosol mass would be distributed if composition diversity had been neglected. Applying the constraint that all particles contain the same volume fraction of each aerosol component (uniform composition), while per-particle BC mass remains the same, causes an artificial redistribution of coating material onto particles containing large amounts of BC. This artificial increase in coating on particles containing most of the $\mathrm{BC}$ mass leads to overestimation of absorption by the particles that contribute most to population-level absorption. As a result, population-level absorption is greater if BC-containing particles are assumed to have identical composition than if the true diversity of the population is represented (Fig. 1e). The negative correlation between the volume fraction of dry coating with the amount of $\mathrm{BC}$ contained in individual particles is consistent with ambient observations $^{36,37}$. This finding suggests that absorption by $\mathrm{BC}$ may be better represented in models that resolve variation in 


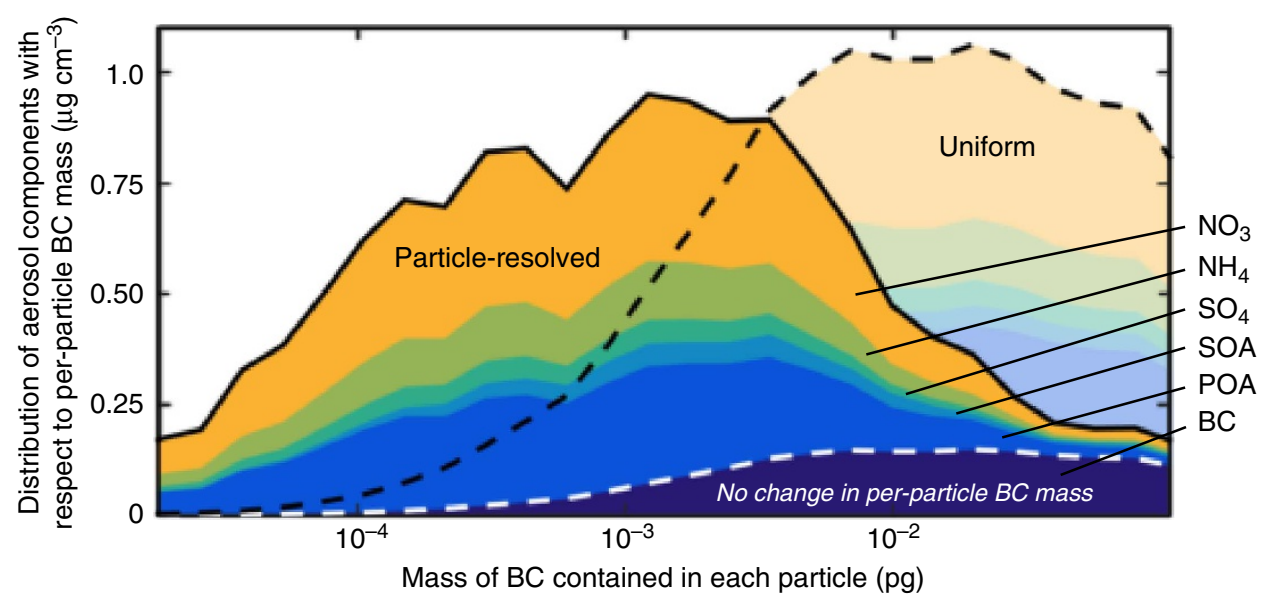

Figure 2 | Mass distribution of aerosol components with respect to per-particle $B C$ mass $\left(\mathbf{d M}_{\mathbf{k}} / \mathbf{d} \log \mathbf{m}_{\mathbf{B C}}\right)$. With particle-resolved composition, most of the $\mathrm{BC}$ mass is contained in particles with large amounts of $\mathrm{BC}$ and small amounts of non- $\mathrm{BC}$ components, or thin coatings. Non- $\mathrm{BC}$ components are $\mathrm{NO}_{3}$ (orange), $\mathrm{NH}_{4}$ (green), $\mathrm{SO}_{4}$ (cyan), $\mathrm{SOA}$ (cerulean) and $\mathrm{POA}$ (blue). If uniform composition is assumed across the same $\mathrm{BC}$ population, the amount of non- $\mathrm{BC}$ material on particles containing large amounts of $\mathrm{BC}$ mass is increased, leading to greater $\mathrm{BC}$ absorption by these particles. Because these particles containing large amounts of $B C$ dominate absorption, population-level absorption enhancement is overpredicted. The total mass of each component for each population of $\mathrm{BC}$-containing particles is the same for both the uniform and particle-resolved cases.

coatings across BC populations (for example, refs 25,38,39), but this potential role of mixing state on $\mathrm{BC}$ absorption should also be validated against observations.

Population-level absorption enhancement. In this section, we describe a nonparametric relationship for absorption enhancement derived from a series of particle-resolved model simulations. The simulations were designed to cover a range of atmospheric conditions, varying from polluted conditions that promote rapid accumulation of coating material to conditions with low gas and particle concentrations that promote slow particle transformations (Methods section). Whereas, Figs 1 and 2 illustrated the importance of composition diversity on absorption enhancement in a single population, here we include thousands of populations, sampled at different time steps from the 100 sensitivity simulations, to determine how absorption enhancement varies with averaged properties of the particle populations. Using a nonparametric regression ${ }^{40,41}$ (Methods section), we developed a relationship for $E_{\mathrm{abs}}$ that depends on variables that many global models already track: (1) the volume fraction of dry aerosol coating that is mixed with BC-containing particles, (2) the hygroscopicity of the coating material and (3) the environmental RH. Including these three variables yielded the best prediction of $E_{\mathrm{abs}}$ in comparison with particle-resolved model results $\left(R^{2}=0.85\right)$. Adding other population-level variables to the regression, such as the refractive index of the coating or the mean diameter of BC inclusions, did not improve the fit.

The relationship between absorption enhancement and the independent variables is shown in Fig. 3. Absorption enhancement is shown as a function of the environmental $\mathrm{RH}$ (horizontal axes) for populations that contain, on average, $50 \%$ dry coating by volume (top panel) and $85 \%$ dry coating by volume (bottom panel), with coatings that are, on average, hydrophobic $\left(\kappa_{\text {coat }}=0.05\right.$, orange $)$ or hygroscopic $\left(\kappa_{\text {coat }}=0.6\right.$, blue $)$, representing organic and inorganic coatings, respectively. This relationship allows the prediction of absorption enhancement for any value of $f_{\text {coat }}$ or $\kappa_{\text {coat }}$, but, for simplicity, only a few combinations of $f_{\text {coat }}$ and $\kappa_{\text {coat }}$ are shown in Fig. 3.

This nonparametric relationship, which uses only populationaveraged composition information, is able to reproduce the population-level absorption enhancement from the particleresolved model with high accuracy (solid lines). Also shown in Fig. 3 is the absorption enhancement that would be predicted if BC-containing particles are assumed to have uniform composition (dashed lines). Differences between the solid and dashed lines in Fig. 3 indicate error in modelled absorption from the simplified representation of particle composition.

For thinly coated particles, such as freshly emitted particles, neglecting composition diversity yields only small error in BC absorption at all relative humidities. Comparison between the top and bottom panels of Fig. 3 demonstrates the effect of coating thickness on absorption enhancement. For the uniform composition treatment (dashed lines), absorption enhancement increases as dry coating material is added to the particles, consistent with previous modelling (for example, refs 14,15) and laboratory measurements(for example, refs 42-44). However, when absorption is modelled at low $\mathrm{RH}$ using particle-resolved composition (solid lines) we find only weak increases in absorption with the amount of coating material, consistent with ambient observations ${ }^{15-17}$. Thus, the finding that a diverse particle population exhibits lower absorption than one with uniform composition holds for particle populations simulated in a wide range of relevant atmospheric conditions.

Although we find $E_{\mathrm{abs}}<1.5$ for diverse populations under dry conditions, absorption increases by as much as $50 \%$ for these populations when particles take up water at high $\mathrm{RH}$. This finding suggests that absorption may be strongly enhanced in humid regions of the atmosphere, even if observations under dry conditions do not capture this enhancement. Ambient particles are dried to $\mathrm{RH} \approx 50 \%$ before absorption measurements, so field studies are unable to observe the response in absorption enhancement to changes in RH. The role of water uptake on absorption enhancement has been demonstrated for cloud droplets that contain $\mathrm{BC}$ through laboratory measurements ${ }^{45}$ and modelling ${ }^{35}$.

Global-scale absorption enhancement. The relationship for absorption enhancement shown in Fig. 3 was applied to global model fields generated by Goddard Institute for Space Studies (GISS) climate modelE ${ }^{19}$ coupled to Multiconfiguration Aerosol Tracker of mIXing state (MATRIX) ${ }^{22}$. MATRIX represents 16 
aerosol modes, 7 of which contain BC (Methods section). Rather than applying the optics calculations from GISS-MATRIX, we applied the relationship shown in Figure 3 that was constructed from thousands of particle-resolved populations. Taking as inputs

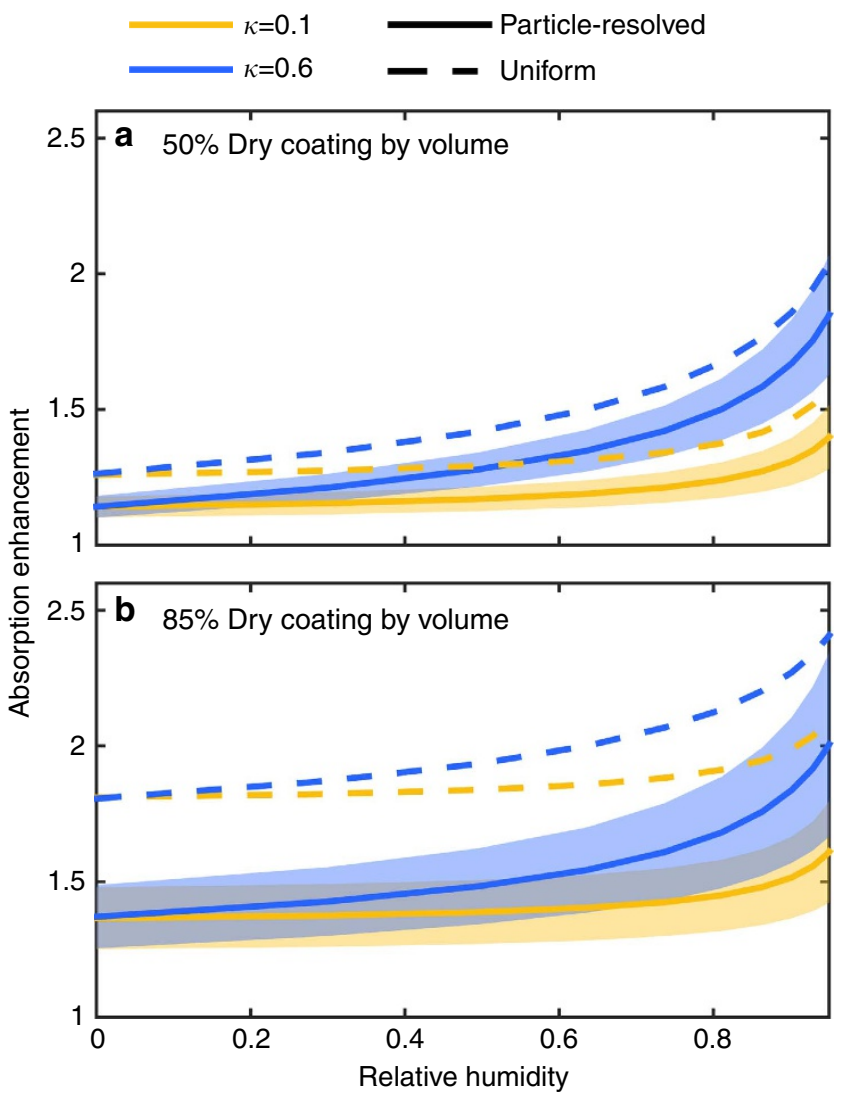

Figure 3 | Absorption enhancement versus environmental relative humidity. Relationship between absorption enhancement (vertical axes) and environmental $\mathrm{RH}$ (horizontal axes) for varying average volume fraction of non-absorbing coatings (panels) and average hygroscopic parameter of the coating material (colours). Population-level absorption computed from particle-resolved composition are shown by solid lines. Shading shows the middle quartiles for the predictions, caused by differences in particle-level composition for populations that have the same population-averaged composition. Absorption enhancement on the basis of particle-resolved composition (solid lines) is compared with absorption enhancement computed from uniform mixture approximation (dashed lines). the $\mathrm{RH}$ in each location, the mass concentration of $\mathrm{BC}$ in each mode and the mass fraction and effective hygroscopicity parameter of dry coating for each mode from GISS-MATRIX, we used the relationship shown in Fig. 3 to estimate the global distribution in absorption enhancement by BC. From the same climate model output, we modelled (1) absorption enhancement for diverse populations but neglecting water uptake (Fig. 4a), (2) absorption enhancement for diverse populations and including water uptake (Fig. 4b) and (3) absorption enhancement for uniform composition and also including water uptake in response to RH (Fig. 4c). Comparison between Fig. 4b,c demonstrates that simplifying the representation of particle composition leads to overestimation of absorption enhancement in many locations (Fig. 4c). Using the relationship that accounts for composition diversity and for water uptake (Fig. 4b), absorption enhancement increases in response to $\mathrm{RH}$, especially over the oceans.

Figure 5 shows the distribution in $\mathrm{BC}$ mass with respect to absorption enhancement level, indicating how frequently BC takes on a particular absorption enhancement level. The dotted, solid and dashed lines in Fig. 5 correspond to the treatments represented in Fig. $4 \mathrm{a}-\mathrm{c}$, respectively. Figure 5 includes BC mass throughout the entire atmosphere, whereas Fig. 4 shows $E_{\mathrm{abs}}$ by BC only at the surface.

Our best estimate of absorption enhancement, accounting for diversity and including aerosol water uptake (solid line in Fig. 5), ranges from $E_{\mathrm{abs}}=1.1$ in some continental areas to $E_{\mathrm{abs}}>2$ in many marine areas, with a $\mathrm{BC}$-weighted mean $E_{\mathrm{abs}}$ of 1.5. This overall absorption enhancement is driven, in part, by enhancement from dry aerosol coatings alone (dotted line in Fig. 5) and further enhancement due to water uptake by the dry coatings (solid line in Fig. 5). If water uptake is ignored, or if it is assumed that water uptake does not contribute to absorption enhancement, the global mean absorption enhancement is underestimated by $5 \%$. On the other hand, if we assume uniform composition among each BC population (dashed line in Fig. 5), the global mean is overestimated of $30 \%$.

\section{Discussion}

Variation in particle composition within BC populations strongly affects modelled absorption. Composition diversity is not captured by aerosol schemes that assume uniform composition across BC populations, but the influence of composition diversity on absorption can be accounted for using a relationship for absorption enhancement on the basis of particle-resolved model results. If particles within a population are assumed to have uniform composition, absorption by $\mathrm{BC}$ under dry conditions can
Accounting for particle-level variation in composition

Assuming uniform comp. across populations

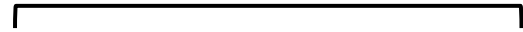

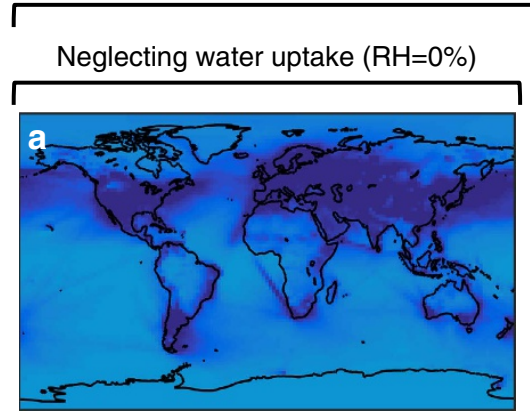

Accounting for variation in water uptake with relative humidity and $\kappa_{\text {coat }}$
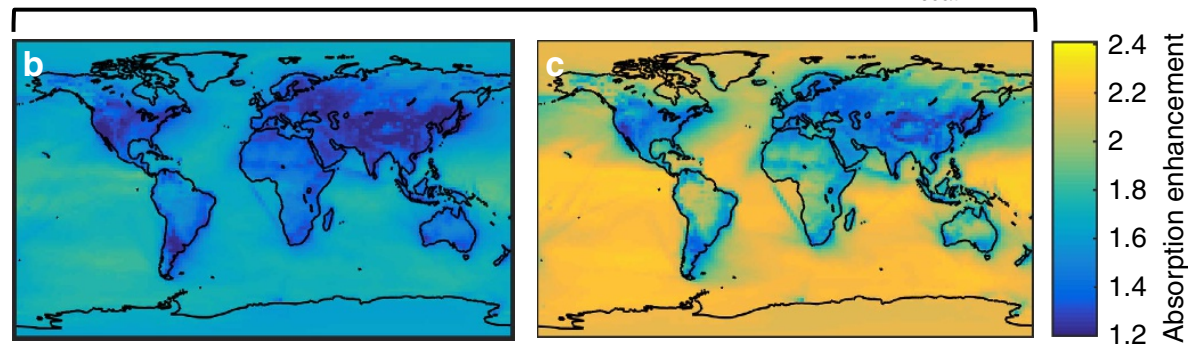

Figure 4 | Spatial variation in absorption enhancement at the surface computed from different representations of particle composition. (a) On the basis of particle-resolved model data, assuming dry conditions (solid lines in Fig. 3, $\mathrm{RH}=0 \%$ ), (b) on the basis of particle-resolved model data, accounting for water uptake (solid lines in Fig. 3, RH from global model) and (c) assuming uniform composition across each BC population and accounting for water uptake (dashed lines in Fig. 3, RH from global model). 


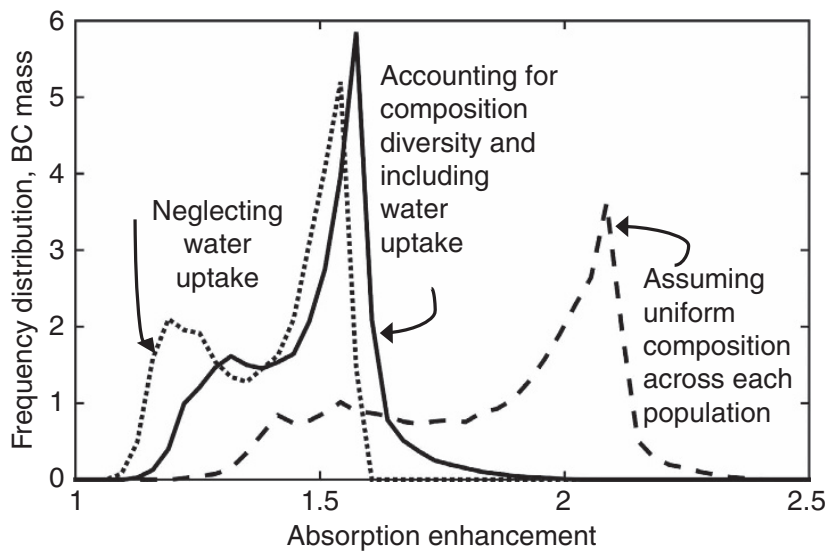

Figure 5 | BC mass density with respect to absorption enhancement for the different representations of particle composition. Solid line shows global distribution in absorption enhancement predicted from most realistic representation, which accounts for water uptake and particle-level variation in composition. Absorption enhancement is underestimated if water uptake is neglected (dotted line) and is overestimated if we assume uniform composition across each BC population (dashed line).

be enhanced by more than factor of two for BC thickly coated with non-absorbing components, consistent with previous modelling ${ }^{14,15}$. If, instead, we account for particle-level variation in composition, we find much weaker levels of absorption enhancement at low $\mathrm{RH}\left(E_{\mathrm{abs}}=1.2-1.5\right)$ and a relatively low sensitivity of $E_{\text {abs }}$ to the amount of coating material, a result that is generally consistent with ambient observations ${ }^{15-17}$. Despite this limited enhancement at low RH, absorption by $\mathrm{BC}$ depends strongly on $\mathrm{RH}$, leading to a factor of two increase in absorption at high RH compared with dry conditions. These model results suggest important roles for diversity in composition and water uptake in determining absorption enhancement that should be confirmed through observations.

\section{Methods}

Simulations with particle-resolved model. The particle-resolved model PartMCMOSAIC tracks the chemical composition of thousands of individual particles as they evolve by condensation of semi-volatile substances, coagulation between particles, particle emissions and dilution with background air (Supplementary Note 1). This study presents simulations of 100 week-long parcel scenarios, which were constructed by sampling a large variable space using latin hypercube sampling ${ }^{46}$ (Supplementary Note 2). Although the simulations include a wide range of ambient conditions, $\mathrm{BC}$ size distributions and the ratio of $\mathrm{BC}$ to $\mathrm{POA}$, which are intended to represent the range of $\mathrm{BC}$ sources and aging conditions, we do not specifically simulate the aerosol evolution in biomass burning plumes. The input parameters that define this variable space are provided in Supplementary Tables 1 and 2 and the resulting range in aerosol concentrations are shown in Supplementary Figs 1 and 2 .

Particle-resolved composition. For each particle, PartMC-MOSIAC tracks the mass of each constituent species $k=1, \ldots, A$, including water. In this study, we extract only dry species from PartMC-MOSAIC, corresponding to $k=1, \ldots, A-1$, and compute the amount water, the $A$ th species, as a post-processing step. Applying the density for each species to the particle-resolved output yields the volume of each species $k$ contained in each particle $i$, denoted $v_{k, i}$. Each particle is then represented by a composition vector $\mathbf{v}_{i}=\left[v_{1, i}, \ldots v_{k, i}, \ldots, v_{A-1, i}\right]$ and the overall dry volume, $V_{\mathrm{d}, i}$, of each particle is given by:

$$
V_{\mathrm{d}, i}=\sum_{k}^{A-1} v_{k, i}
$$

Variability in the volume fraction and hygroscopicity of coating thickness for the baseline population are shown in Supplementary Figs 3 and 4, respectively.
Uniform mixture approximation. Under the uniform mixture approximation, each particle is represented by the vector $\tilde{\mathbf{v}}_{i}$. The volume of each species $k$ in each particle $i$ is adjusted such that the volume fraction of each dry component is the same for all particles, while the volume of BC in each particle $\left(v_{\mathrm{b}, i}\right)$ is the same as in the particle-resolved representation:

$$
\tilde{v}_{k, i}=v_{\mathrm{b}, i} \frac{\sum_{j}^{N_{\mathrm{BC}}} v_{k, j}}{\sum_{j}^{N_{\mathrm{BC}}} v_{\mathrm{b}, j}}
$$

The overall dry volume under the particle-resolved representation, $\tilde{V}_{\mathrm{d}, i}$, is computed from equation 1, but using the composition vector $\tilde{\mathbf{v}}_{k, i}$ rather than $\mathbf{v}_{k, i}$. The value for $\tilde{V}_{\mathrm{d}, i}$ varies between particles and, for each particle, also differs from the value of $V_{\mathrm{d}, i}$.

Water uptake modelled with $\boldsymbol{\kappa}$-Köhler. The wet volume $V_{i}$ is computed for each particle at each environmental RH using the $\kappa$-Köhler model ${ }^{33}$ :

$$
\frac{\mathrm{RH}}{\exp \left(A V_{i}^{-1 / 3}\right)}=\frac{V_{i}-V_{\mathrm{d}, i}}{V_{i}-V_{\mathrm{d}, i}\left(1-\kappa_{i}\right)},
$$

where $V_{\mathrm{d}, i}$ is the volume of dry aerosol components contained in particle $i$ and $\kappa_{i}$ is its hygroscopicity parameter. The hygroscopicity parameter $\kappa_{i}$ is computed as the volume-weighted average of $\kappa$ for each aerosol species:

$$
\kappa_{i}=\frac{1}{V_{\mathrm{d}, i}} \sum_{k}^{A-1} v_{k, i} \kappa_{k} .
$$

Similarly, the effective hygroscopicity corresponding to the average composition, denoted $\tilde{\kappa}_{i}$, is computed from equation 4 but using the composition from the uniform mixture approximation, $\tilde{\mathbf{v}}_{i}$. Values for $\kappa$ for each species are provided in Supplementary Table 2 . The value of $A$ depends on environmental variables and properties of water, as shown in Supplementary Equation 1.

Absorption modelled with DEMA-Mie. The absorption cross-section $\sigma_{\mathrm{abs}, i}$ of each particle $i$ is computed using Mie theory, treating each BC-containing particle as an effective medium using the DEMA ${ }^{31,32}$. The Mie model takes as inputs the effective relative permittivity $\epsilon_{i}$, which is computed from DEMA, and the wet volume $V_{i}$. The effective relative permittivity $\epsilon_{i}$ for each particle $i=1, \ldots, N_{\mathrm{BC}}$ is computed from the following non-linear equation:

$$
\begin{array}{r}
\left(1-\frac{v_{\mathrm{b}, i}}{V_{i}}\right) \frac{\epsilon_{\mathrm{c}, i}-\epsilon_{i}}{\epsilon_{\mathrm{c}, i}-2 \epsilon_{i}}+\left(\frac{V_{\mathrm{b}, i}}{V_{i}}\right) \frac{\epsilon_{\mathrm{b}, i}-\epsilon_{i}}{\epsilon_{\mathrm{b}, i}-2 \epsilon_{i}}+ \\
\frac{2 \pi}{45}\left(\frac{2}{\lambda}\right)^{2}\left(\epsilon_{\mathrm{b}, i}-\epsilon_{i}\right)\left[1+\frac{5 \epsilon_{i}}{2 \epsilon_{\mathrm{b}, i}+3 \epsilon_{i}}+\right. \\
\left.\frac{18 \epsilon_{i}\left(\epsilon_{\mathrm{b}, i}-2 \epsilon_{i}\right)}{\left(\epsilon_{\mathrm{b}, i}+2 \epsilon_{i}\right)^{2}}\right] \int r^{2} n_{i}(r) \mathrm{d} r=0,
\end{array}
$$

which depends on the total volume of each particle $\left(V_{i}\right)$, the volume of BC contained in each particle $\left(V_{\mathrm{b}, i}\right)$, the relative permittivity of $\mathrm{BC}\left(\epsilon_{\mathrm{b}, i}\right)$, the relative permittivity of each particle's non-BC coating $\left(\epsilon_{c, i}\right)$ and the number and size of BC inclusions $(n(r))$. The relative permittivity of the coating material, $\epsilon_{\mathrm{c}, i}$ is computed as the volume-weighted average of the non-BC components, including water. The absorption cross-section of particles under the uniform mixture approximation, $\tilde{\sigma}_{\text {abs }, i}$, is computed in a similar manner, but using $\tilde{V}_{i}$ rather than $V_{i}$ in equation 5. See Supplementary Note 3.

Enhancement in light absorption by $\mathrm{BC}$ within an individual particle $i$ is then given by:

$$
E_{\mathrm{abs}, i}=\frac{\sigma_{\mathrm{abs}, i}}{\sigma_{\mathrm{core}, \mathrm{abs}, i}} .
$$

Absorption enhancement at the population-level, considering the all $\mathrm{BC}$ containing particles, is computed as:

$$
E_{\mathrm{abs}}=\frac{\sum_{i}^{N_{\mathrm{BC}}} \sigma_{\mathrm{abs}, i}}{\sum_{i}^{N_{\mathrm{BC}}} \sigma_{\mathrm{abs}, \text { core }, i}},
$$

where $\sigma_{\mathrm{abs}, i}$ is the absorption coefficient of each BC-containing particle $i=1, \ldots, N_{\mathrm{BC}}$, and $\sigma_{\mathrm{abs}, \text { core }, i}$ is the absorption coefficient by the same amount of BC but as a single, uncoated core.

Similarly, population-level absorption enhancement under the uniform mixture approximation is computed from the sum over $\tilde{\sigma}_{\mathrm{abs}, i}$ for all particles in the population:

$$
\tilde{E}_{\mathrm{abs}}=\frac{\sum_{i}^{N_{\mathrm{BC}}} \tilde{\sigma}_{\mathrm{abs}, i}}{\sum_{i}^{N_{\mathrm{BC}}} \sigma_{\mathrm{abs}, \text { core }, i}},
$$

Kernel density regression. The nonparametric relationship for $E_{\text {abs }}$ shown in Fig. 3 was produced using a kernel density regression ${ }^{40,41}$ on model data from thousands of particle-resolved model populations. Populations were sampled from the 100 scenarios to represent $\mathrm{BC}$-containing particles that had aged to varying 
degree and under a variety of conditions. The regression analysis was used to identify the set of independent variables that most affect $E_{\text {abs }}$, as described in ref 47 and to construct the relationship for $E_{\text {abs }}$ shown in Fig. 3 (Supplementary Note 4 and Supplementary Figure 5).

Global climate model GISS-MATRIX. MATRIX is an aerosol scheme based on the quadrature method of moments ${ }^{48}$ that represents 16 aerosol modes. Operating within the general circulation climate modelE ${ }^{19}$, MATRIX tracks two moments of each aerosol distribution, number and mass, assuming lognormal size distributions for emissions and removal calculations. MATRIX includes sulfate, nitrate, ammonium, aerosol water, $\mathrm{BC}, \mathrm{POA}$, mineral dust and sea salt and simulates nucleation, secondary aerosol formation, condensation and coagulation. The climate simulations discussed here are identical to ref. 49. The distribution in the volume fraction of dry coating on $\mathrm{BC}$, hygroscopicity of dry coating and $\mathrm{RH}$ are shown for surface grid cells in Supplementary Figure 6.

Data availability. PartMC version 2.1.5 was used for the simulations in this paper, available at: http://lagrange.mechse.illinois.edu/partmc/. MOSAIC is available from Rahul Zaveri. The simulation settings are outlined in Supplementary Tables 1 and 2, and the input files are available from Laura Fierce. Code for modelling water uptake with $\kappa$-Köhler and absorption with DEMA are available from Laura Fierce. Model data simulated with GISS-MATRIX are available from Susanne Bauer.

\section{References}

1. Rosen, H., Hansen, A., Gundel, L. \& Novakov, T. Identification of the optically absorbing component in urban aerosols. Appl. Opt. 17, 3859-3861 (1978).

2. Schwarz, J. et al. Measurement of the mixing state, mass, and optical size of individual black carbon particles in urban and biomass burning emissions. Geophys. Res. Lett. 35, L13810 (2008).

3. Liu, D. et al. Ambient black carbon particle hygroscopic properties controlled by mixing state and composition. Atmos. Chem. Phys. Discuss. 12, 28955-28992 (2012).

4. Healy, R. M. et al. Single particle diversity and mixing state measurements. Atmos. Chem. Phys. 14, 6289-6299 (2014).

5. Sodeman, D. A., Toner, S. M. \& Prather, K. A. Determination of single particle mass spectral signatures from light-duty vehicle emissions. Environ. Sci. Technol. 39, 4569-4580 (2005).

6. Toner, S. M., Sodeman, D. A. \& Prather, K. A. Single particle characterization of ultrafine and accumulation mode particles from heavy duty diesel vehicles using aerosol time-of-flight mass spectrometry. Environ. Sci. Technol. 40, 3912-3921 (2006).

7. Maricq, M. M. Chemical characterization of particulate emissions from diesel engines: a review. J.Aerosol Sci. 38, 1079-1118 (2007)

8. Healy, R. et al. Sources and mixing state of size-resolved elemental carbon particles in a European megacity: Paris. Atmos. Chem. Phys. 12, 1681-1700 (2012).

9. Weingartner, E., Burtscher, H. \& Baltensperger, U. Hygroscopic properties of carbon and diesel soot particles. Atmos. Environ. 31, 2311-2327 (1997)

10. Whiteaker, J. R., Suess, D. T. \& Prather, K. A. Effects of meteorological conditions on aerosol composition and mixing state in Bakersfield, CA. Environ. Sci. Technol. 36, 2345-2353 (2002).

11. Johnson, K. et al. Processing of soot in an urban environment: case study from the Mexico City Metropolitan Area. Atmos. Chem. Phys. 5, 3033-3043 (2005).

12. Wang, J., Cubison, M., Aiken, A., Jimenez, J. \& Collins, D. The importance of aerosol mixing state and size-resolved composition on CCN concentration and the variation of the importance with atmospheric aging of aerosols. Atmos. Chem. Phys. 10, 7267-7283 (2010).

13. Cheng, Y. et al. Size-resolved measurement of the mixing state of soot in the megacity Beijing, China: diurnal cycle, aging and parameterization. Atmos. Chem. Phys. 12, 4477-4491 (2012).

14. Chung, C. E., Lee, K. \& Müller, D. Effect of internal mixture on black carbon radiative forcing. Tellus B 64, 10925 (2011).

15. Cappa, C. D. et al. Radiative absorption enhancements due to the mixing state of atmospheric black carbon. Science 337, 1078-1081 (2012).

16. Liu, S. et al. Enhanced light absorption by mixed source black and brown carbon particles in UK winter. Nat. Commun. 6, 8435 (2015).

17. Healy, R. et al. Light-absorbing properties of ambient black carbon and brown carbon from fossil fuel and biomass burning sources. J. Geophys. Res. Atmos. 120, 6619-6633 (2015).

18. Chin, M. et al. Multi-decadal aerosol variations from 1980 to 2009: a perspective from observations and a global model. Atmos. Chem. Phys. 14, 3657-3690 (2012).

19. Schmidt, G. A. et al. Configuration and assessment of the GISS ModelE2 contributions to the CMIP5 archive. J. Adv. Model. Earth Syst. 6, 141-184 (2014).
20. Binkowski, F. \& Roselle, S. Models-3 Community Multiscale Air Quality (CMAQ) model aerosol component: 1. Model description. J. Geophys. Res. 108, 15 (2003).

21. Stier, P. et al. The aerosol-climate model ECHAM5-HAM. Atmos. Chem. Phys 5, 1125-1156 (2005).

22. Bauer, S. E. et al. MATRIX (Multiconfiguration Aerosol TRacker of mIXing state): an aerosol microphysical module for global atmospheric models. Atmos. Chem. Phys. 8, 6003-6035 (2008).

23. Jacobson, M. Development and application of a new air pollution modeling system-II. Aerosol module structure and design. Atmos. Environ. 31, 131-144 (1997).

24. Adams, P. J. \& Seinfeld, J. H. Predicting global aerosol size distributions in general circulation models. J. Geophys. Res. Atmos. 107, 4370 (2002).

25. Jacobson, M. Z. Analysis of aerosol interactions with numerical techniques for solving coagulation, nucleation, condensation, dissolution, and reversible chemistry among multiple size distributions. J. Geophys. Res. Atmos. 107, 4366 (2002).

26. Adachi, K., Chung, S. H. \& Buseck, P. R. Shapes of soot aerosol particles and implications for their effects on climate. J. Geophys. Res. Atmos. 115, D15206 (2010).

27. Kahnert, M., Nousiainen, T., Lindqvist, H. \& Ebert, M. Optical properties of light absorbing carbon aggregates mixed with sulfate: assessment of different model geometries for climate forcing calculations. Opt. Express 20, 10042-10058 (2012).

28. Scarnato, B. V., Vahidinia, S., Richard, D. T. \& Kirchstetter, T. W. Effects of internal mixing and aggregate morphology on optical properties of black carbon using a discrete dipole approximation model. Atmos. Chem. Phys. 13, 5089-5101 (2013).

29. Riemer, N., West, M., Zaveri, R. \& Easter, R. Simulating the evolution of soo mixing state with a particle-resolved aerosol model. J. Geophys. Res. 114, D09202 (2009)

30. Zaveri, R., Easter, R., Fast, J. \& Peters, L. Model for Simulating Aerosol Interactions and Chemistry (MOSAIC). J. Geophys. Res. 113, D13204 (2008).

31. Chylek, P., Ramaswamy, V. \& Cheng, R. J. Effect of graphitic carbon on the albedo of clouds. J. Atmos. Sci. 41, 3076-3084 (1984).

32. Jacobson, M. Z. Effects of externally-through-internally-mixed soot inclusions within clouds and precipitation on global climate. J. Phys. Chem. A 110, 6860-6873 (2006).

33. Petters, M. D. \& Kreidenweis, S. M. A single parameter representation of hygroscopic growth and cloud condensation nucleus activity. Atmos. Chem. Phys. 7, 1961-1971 (2007).

34. Aquila, V. et al. MADE-in: a new aerosol microphysics submodel for global simulation of insoluble particles and their mixing state. Geosci. Model Dev. 4, 325-355 (2011).

35. Jacobson, M. Z. Investigating cloud absorption effects: Global absorption properties of black carbon, tar balls, and soil dust in clouds and aerosols. J. Geophys. Res. Atmos. 117, D06205 (2012).

36. Metcalf, A. et al. Black carbon aerosol over the Los Angeles basin during CalNex. J. Geophys. Res. Atmos. 117, D00V13 (2012).

37. Sedlacek, A. J., Lewis, E. R., Kleinman, L., Xu, J. \& Zhang, Q. Determination of and evidence for non-core-shell structure of particles containing black carbon using the Single-Particle Soot Photometer (SP2). Geophys. Res. Lett. 39, L06802 (2012).

38. Oshima, N. et al. Aging of black carbon in outflow from anthropogenic sources using a mixing state resolved model: Model development and evaluation. J. Geophys. Res. Atmos. 114, D18202 (2009).

39. Matsui, H. et al. Development and validation of a black carbon mixing state resolved three-dimensional model: Aging processes and radiative impact. J. Geophys. Res. Atmos. 118, 2304-2326 (2013).

40. Nadaraya, E. A. On estimating regression. Theory Probab.Appl. 9, 141-142 (1964).

41. Watson, G. S. Smooth regression analysis. Sankhyā Indian J. Stat. Series A $\mathbf{2 6}$, 359-372 (1964)

42. Schnaiter, M. et al. Absorption amplification of black carbon internally mixed with secondary organic aerosol. J. Geophys. Res. 110, D19204 (2005).

43. Cross, E. S. et al. Soot particle studies-instrument inter-comparison-project overview. Aerosol Sci. Technol. 44, 592-611 (2010).

44. Shiraiwa, M., Kondo, Y., Iwamoto, T. \& Kita, K. Amplification of light absorption of black carbon by organic coating. Aerosol Sci. Technol. 44, 46-54 (2010).

45. Mikhailov, E. F., Vlasenko, S. S., Podgorny, I. A., Ramanathan, V. \& Corrigan, C. E. Optical properties of soot-water drop agglomerates: An experimental study. J. Geophys. Res.Atmos. 111, D07209 (2006).

46. McKay, M. D., Beckman, R. J. \& Conover, W. J. Comparison of three methods for selecting values of input variables in the analysis of output from a computer code. Technometrics 21, 239-245 (1979).

47. Fierce, L., Riemer, N. \& Bond, T. C. Explaining variance in black carbon's aging timescale. Atmos. Chem. Phys. 15, 3173-3191 (2015). 
48. McGraw, R. Description of aerosol dynamics by the Quadrature Method of Moments. Aerosol Sci. Technol. 27, 255-265 (1997).

49. Bauer, S. E. \& Menon, S. Aerosol direct, indirect, semidirect, and surface albedo effects from sector contributions based on the IPCC AR5 emissions for preindustrial and present-day conditions. J. Geophys. Res. Atmos. 117, D01206 (2012).

\section{Acknowledgements}

This work was supported by the US Environmental Protection Agency (R83504201) and by NASA (NNX09AK66G). F. Mena was funded by the Department of

Energy under DE-FG02-08ER64533 and by a Fulbright-Chile CONICYT fellowship. L. Fierce is funded by a NOAA Climate \& Global Change Postdoctoral Fellowship through the University Corporation for Atmospheric Research Visiting Scientists Program.

\section{Author contributions}

L. Fierce designed the study, performed the particle-resolved model simulations, analyzed the model data and wrote the paper. T. C. Bond and N. Riemer were involved in the study design, the interpretation of the results and the manuscript preparation. S. E. Bauer performed global model simulations with GISS-MATRIX. F. Mena assisted in modelling particle absorption with DEMA.

\section{Additional information}

Supplementary Information accompanies this paper at http://www.nature.com/ naturecommunications

Competing financial interests: The authors declare no competing financial interests.

Reprints and permission information is available online at http://npg.nature.com/ reprintsandpermissions/

How to cite this article: Fierce, L. et al. Black carbon absorption at the global scale is affected by particle-scale diversity in composition. Nat. Commun. 7:12361 doi: 10.1038/ ncomms12361 (2016).

\section{(c) (1)}

This work is licensed under a Creative Commons Attribution 4.0 International License. The images or other third party material in this article are included in the article's Creative Commons license, unless indicated otherwise in the credit line; if the material is not included under the Creative Commons license, users will need to obtain permission from the license holder to reproduce the material. To view a copy of this license, visit http://creativecommons.org/licenses/by/4.0/

(C) The Author(s) 2016 